\title{
Intraspinal psammomatous melanotic schwannoma not associated with Carney complex: case report
}

\author{
Saman Shabani, MD,, ${ }^{1,3}$ Susan M. Fiore, MS, ${ }^{1}$ Roberta Seidman, MD, ${ }^{2}$ and Raphael P. Davis, MD ${ }^{1}$ \\ 1Department of Neurological Surgery and the Neurosciences Institute, and 'Department of Pathology, Stony Brook University, \\ Stony Brook, New York; and ${ }^{3}$ Department of Neurological Surgery, Medical College of Wisconsin, Milwaukee, Wisconsin
}

\begin{abstract}
The authors present a case of intraspinal malignant psammomatous melanotic schwannoma (PMS) not associated with Carney complex and review all reported cases not associated with this syndrome. The focus of this review paper is on the characteristics of the malignant progression of PMS.
\end{abstract}

\begin{abstract}
A 54-year-old man had a history of squamous cell carcinoma of the neck and tonsillar carcinoma. The patient's serial CT scanning study showed a mass in the left C-5 foramen. On presentation he was neurologically intact. After 18 months, the patient developed radiating pain down the left arm with decreased sensation. MRI of the cervical spine showed an enhancing $2.1 \times 1.5 \times 1.9-\mathrm{cm}$ mass in the left C5-6 foramen. A C5-6 hemilaminectomy was performed with gross-total removal of the tumor. At 3 months postoperatively, the patient developed new-onset pain and weakness. MRI showed a dumbbell-shaped mass in the left C-7 foramen. MRI of the pelvis showed a $1.4 \times 1.0-\mathrm{cm}$ lesion on the right ischium and a $1.1 \times 2.8-\mathrm{cm}$ lesion on the right inferior pubic ramus. Anterior cervical discectomy of C5-6 and C6-7 with corpectomy of C-6 with subtotal resection of the tumor was completed. PMS should not be considered a benign tumor because in $41.1 \%$ of patients, including the patient in this report, the tumor progresses to malignancy. Long-term follow-up is needed in these patients. New surgical treatment plans should be considered.
\end{abstract}

http://thejns.org/doi/abs/10.3171/2014.11.SPINE13990

KEY WORDS psammomatous melanotic schwannoma; intraspinal; Carney complex; oncology

$\mathrm{P}$ SAMmomatous melanotic schwannoma (PMS) is a rare tumor and in $50 \%$ of cases is a component of Carney complex, an inherited autosomal-dominant disorder. ${ }^{3,4}$ The biological behavior of this tumor is unclear because there have been few cases of PMS, and even lesions considered benign without mitosis, atypia, or necrosis may present with recurrence and/or metastasis after many years. In this paper, we report a case of PMS and review the literature of reported intraspinal PMS cases not associated with Carney complex. To our knowledge, no report has been published selectively to look at the characteristics of intraspinal PMS not associated with Carney complex.

\section{Case Report}

History and Examination

This 54-year-old man had a medical history significant for squamous cell carcinoma of the neck and tonsillar carcinoma after resection and radiation therapy. On serial CT scanning of the patient's head and neck, there was an incidental finding of a left C-5 foraminal mass. The mass on CT scanning with contrast showed a well-defined ovoid enhancing lesion in the left C-5 foramen measuring 1.9 $\mathrm{cm}($ axial $) \times 1.1 \mathrm{~cm}$ (anteroposterior) $\times 1.4 \mathrm{~cm}$ (craniocaudal). There was displacement of the adjacent left vertebral artery, smooth erosion of the anterior margin of the adjacent vertebral facet, and enlargement of the anterolateral portion of the foramen. MRI with and without gadolinium contrast of the neck showed an ovoid enhancing mass in the left C5-6 neural foramen with smooth scalloping of the neural foramen. The mass measured approximately $1.9 \times 1.3 \mathrm{~cm}$. There was patchy marrow edema in the left aspect of the C5-7 vertebral bodies and adjacent soft tissues. There was also additional enhancement in the left C6-7 foramen. MRI with and without gadolinium contrast

ABBREVIATIONS MRA = MR angiography; PMS = psammomatous melanotic schwannoma.

SUBMITTED November 7, 2013. ACCEPTED November 6, 2014.

INCLUDE WHEN CITING Published online May 15, 2015; DOI: 10.3171/2014.11.SPINE13990.

DISCLOSURE The authors report no conflict of interest concerning the materials or methods used in this study or the findings specified in this paper. The Department of Neurological Surgery at Stony Brook Medicine provided funding. 
of the cervical spine showed enhancing soft tissue within the left neural foramen with intermixed foci of low signal measuring approximately $2.3 \mathrm{~cm}$ (axial) $\times 1.4 \mathrm{~cm}$ (anteroposterior) $\times 1.5 \mathrm{~cm}$ (craniocaudal). The mass resulted in anteromedial displacement of the left vertebral artery. There was also a questionable smaller enhancing lesion in the left C6-7 neural foramen.

On physical examination during the first office visit, there were no palpable masses, and the patient had a good cervical range of motion in the cervical spine. The patient had Grade 5/5 strength on motor examination in all muscle groups tested, intact sensory examination to light touch, Grade 2+ bilateral reflexes, and unremarkable gait. The patient was offered surgical intervention but elected to undergo follow-up with serial MRI performed every 6 months. After consecutive follow-up visits with stable findings on imaging, the 6-month follow-up interval was changed to yearly. Throughout the follow-up the patient was symptom free with intact sensation and Grade 5/5 motor strength.

After 18 months of follow-up, the patient developed significant pain radiating from the left cervical region into the left thumb and index finger. The pain was constant and awakened the patient from sleep at night. He tried antiinflammatory and muscle relaxants, which did not decrease the severity of the pain. He started taking oxycodone prior to sleep, which only gave him approximately $2-3$ hours of relief. On physical examination, his strength was Grade $5 / 5$ in the upper extremities in both proximal and distal muscle groups. However, sensation was diminished to light touch in the left index finger and thumb. His deep tendon reflexes were hypoactive. MRI of the cervical spine with and without contrast and MR angiography (MRA) of the head and neck without contrast were performed. Cervical MRI showed a heterogeneous enhancing mass in the left neural foramen at C5-6 with benign remodeling of adjacent bone, resulting in widening of the neural foramen. The mass had imaging characteristics consistent with a nerve sheath tumor. The mass measurements were $2.1 \mathrm{~cm}$ (axial) $\times 1.5 \mathrm{~cm}$ (anteroposterior) $\times 1.9 \mathrm{~cm}$ (craniocaudal) (Fig. 1). The vertebral artery was displaced anteriorly by the anterior border of the mass (Fig. 1). However, it had normal flow signal and enhancement. MRA of the head
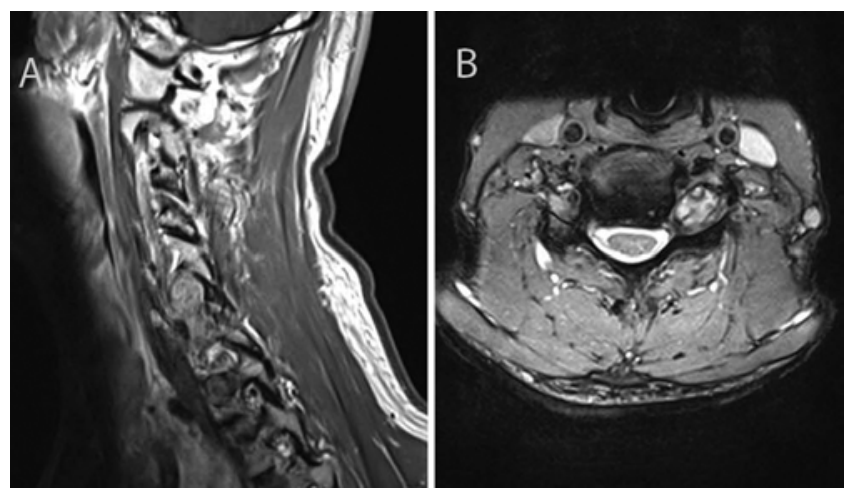

FIG. 1. MR images obtained before the first surgery. A: Sagittal T1weighted FLAIR postcontrast image showing the mass in the neural foramen. B: Axial T2-weighted image showing a heterogeneous mass in the left neural foramen at C5-6. The mass has caused anterior displacement of vertebral artery. did not show any evidence of vasculopathy. MRA of the neck showed a mass within the left neural foramen at C56 , which displaced the left vertebral artery anteromedially. However, the artery was patent with normal flow-related signal. Due to his C- 6 radiculopathy, the patient agreed to undergo a C5-6 hemilaminectomy.

\section{First Operation}

The patient underwent a hemilaminectomy of C5-6. The tumor was highly vascularized and arose from the dorsal root with a small component at the root entry zone. This necessitated opening and ultimately excision of the dural root sheath and the violation of the subarachnoid space. Because the tumor was presumed to be schwannoma based on the preoperative images, it was coagulated and then opened to be internally decompressed using the Cavitron Ultrasonic Surgical Aspirator (CUSA). Upon opening the tumor, the contents were grossly melanotic. The melanin bodies and the tumor capsule helped in differentiating the tumor margins from the normal structures. Complete removal was achieved by removing the capsule and the melanin-containing tumor.

\section{First Pathological Examination}

The intradural well-circumscribed tumor showed heavy melanin pigmentation in both the intracytoplasmic and extracellular regions. The neoplasm was composed of spindle and epithelioid cells (Fig. 2) with atypical nuclei that possessed prominent nucleoli and scattered intranuclear cytoplasmic inclusions. Immunohistochemical analyses for S100, HMB-45/Melan-A cocktail, and Ki 67 were done. The neoplasm showed extensive cytoplasmic and some nuclear labeling of S100 (Fig. 2). HMB-45/Melan-A and Ki 67 were negative. A $B R A F$ mutation study was done. However, the assay-specific requirement to generate a result was not met, which could have been due to either nucleic acid concentration below the detectable limits or factors that influenced the integrity of the specimen.

\section{Postoperative Course}

Three months after resection of the tumor, the patient developed new onset of pain, mild weakness in the left upper extremity primarily affecting raising his hand above his head, and numbness in his middle finger on the left side. CT and MRI of the cervix both with contrast showed an enhancing soft mass within the lateral recess of C5-6 extending out the neural foramen. The mass extended lateral to the vertebral artery at the site of previous tumor (Fig. 3A). Moreover, a dumbbell-shaped mass was noticed on MRI in the left C-7 foramen. PET and whole-body bone scanning showed significant uptake in the C5-6 vertebrae. CT scanning of the chest, abdomen, and pelvis without contrast showed a 7-mm lytic lesion in the right ischium and a $12-\mathrm{mm}$ lytic lesion in the right inferior pubic ramus. MRI of the pelvis with and without contrast showed a 1.4 $\times 1.0-\mathrm{cm}$ lesion on the right ischium and a $1.1 \times 2.8-\mathrm{cm}$ lesion on the right inferior pubic ramus. The pelvic biopsy was PMS.

\section{Second Operation}

Anterior cervical discectomy of C5-6 and C6-7 with 


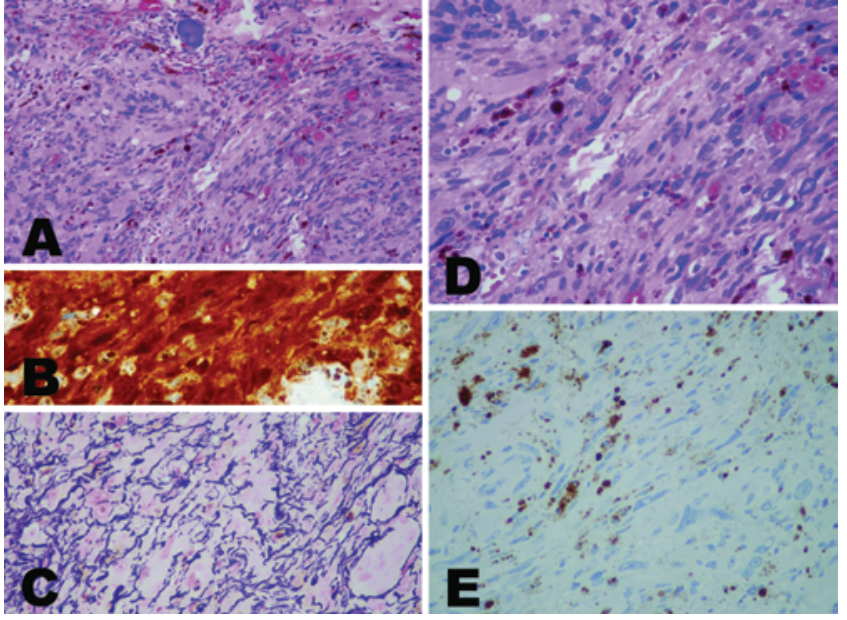

FIG. 2. The original neoplasm, a circumscribed intradural mass attached to the left C-6 nerve root, from the first surgery. A: Low-power image of an $\mathrm{H} \& \mathrm{E}$-stained section showing a largely spindle cell neoplasm with brown melanin pigmentation distributed throughout the lesion. A psammoma body is seen as a blue well-demarcated structure at the upper center of the image. B: S100 immunohistochemistry demonstrating labeling of nuclei and cytoplasm of the neoplastic cells. The unstained structure in the lower right quadrant of the image is a blood vessel. C: Reticulin stain demonstrating abundant pericellular reticulin deposition, seen as a black threadlike material. This finding is highly characteristic of schwannoma and would not be expected in melanoma. D: Highpower detail of a portion of the field seen in panel A, demonstrating ovoid, elongated nuclei, some with small nucleoli and no mitotic figures. Melanin pigment is visible within the cytoplasm of many of the neoplastic cells. E: Immunohistochemistry for the Ki 67 proliferation marker demonstrating labeling of only a rare nucleus in this field, indicated by the round, nucleus-shaped brown labeling, not to be confused with the dustlike and irregularly aggregated cytoplasmic melanin pigment. Original magnification $\times 100(A) ; \times 400(B-E)$.

corpectomy of C-6 with resection of tumor involving the bone and epidural space was performed. The tumor could not be removed completely due to a previous posterior surgical approach and attachment of tumor to the ligament, which could not be removed easily (Fig. 3B). In addition, the tumor extended laterally into the epidural space, which was not approachable anteriorly. The patient died 4 months after the second operation.

\section{Second Pathology}

The pathology was recurrent/metastatic PMS. The neoplasm showed atypical spindle and epithelioid cells and focally prominent cytoplasmic pigmentation (Fig. 4). The tumor was morphologically similar to the previous tumor. However, mitotic figures and local areas of necrosis were identifiable. The Ki 67 labeling index varied from region to region, but an estimated $10 \%-20 \%$ of the nuclei were positive (Fig. 4). Melan-A was positive in one-third to onehalf of the neoplastic cells, and HMB-45 was positive in the majority of the cells. BRAF codon 600 mutation was negative, and reticulin stain showed little reticulin deposition.

\section{Discussion}

PMS is a rare melanin-producing tumor that contains psammoma bodies. About 55\% of these cases are sporad-
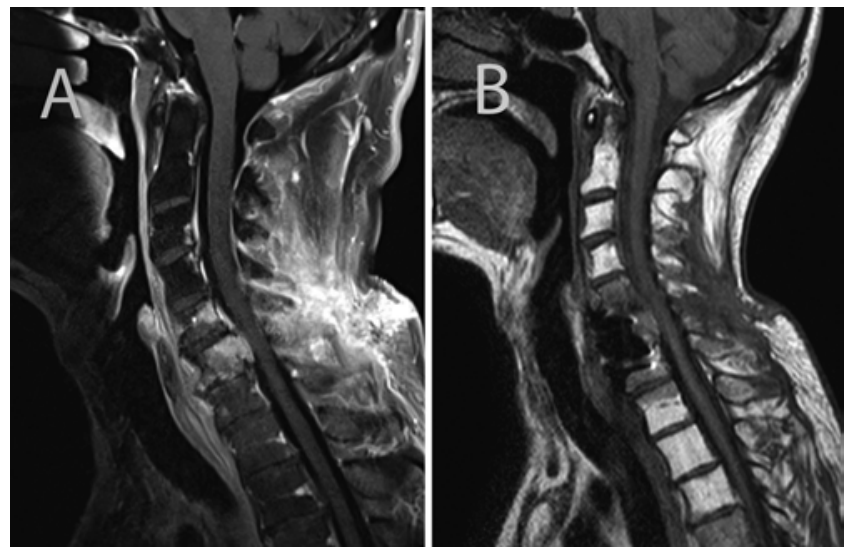

FIG. 3. A: Sagittal T1-weighted FLAIR postoperative MR image obtained 3 months after the first surgery. There is metastasis and recurrence of the tumor. B: Sagittal T1-weighted postoperative MR image obtained on the day of the second surgery. There is incomplete resection of tumor due to previous surgery and extension of it into the epidural space, which was not accessible anteriorly.

ic, and the rest are associated with Carney complex. ${ }^{3}$ The first case was reported by Miller in 1932, ${ }^{14}$ and in 1961 Hodson $^{6}$ reported PMS affecting cranial nerves, alveolar nerves, palate, parotid gland, and neck. In 1985, Carney and colleagues ${ }^{4}$ described the autosomal-dominant syndrome of Carney complex.

Melanotic schwannomas are generally derived from peripheral nerves and sympathetic ganglions, but extraneural locations have been reported. The gross pathology shows a melanin-containing tumor that appears as round to ovoid, is several centimeters in diameter, and is gray to black.17 Most of these tumors are not encapsulated. ${ }^{17}$ Histologically,

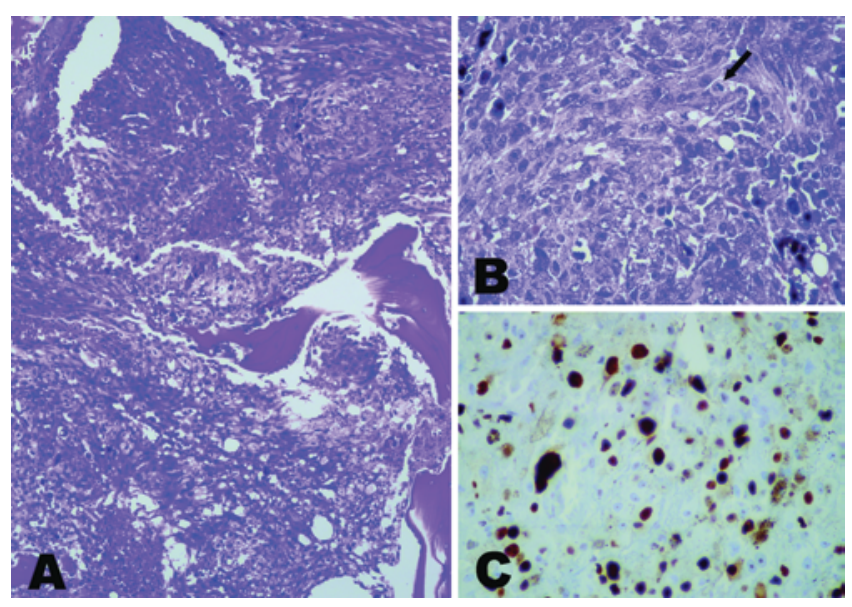

FIG. 4. Immunohistochemical analysis of the C-6 vertebral body lesion from the second surgical procedure. A: $\mathrm{H} \& \mathrm{E}$-stained section at low power showing a spindled, focally epithelioid pigmented neoplasm infiltrating and destroying bone. B: $\mathrm{H} \& \mathrm{E}$-stained section at higher power showing that there are more nucleoli and they are larger than those in the first surgical specimen. A mitotic figure is present in the upper right quadrant of the image, indicated by an arrow. C: Immunohistochemistry for the Ki 67 proliferation marker demonstrates significantly greater nuclear labeling than the specimen from the first surgery. The nuclear labeling is the dense uniform brown labeling in the shape of nuclei, in contrast to the particulate appearance of the brown melanin pigment. Original magnification $\times 100(A) ; \times 400(B$ and $C)$. 


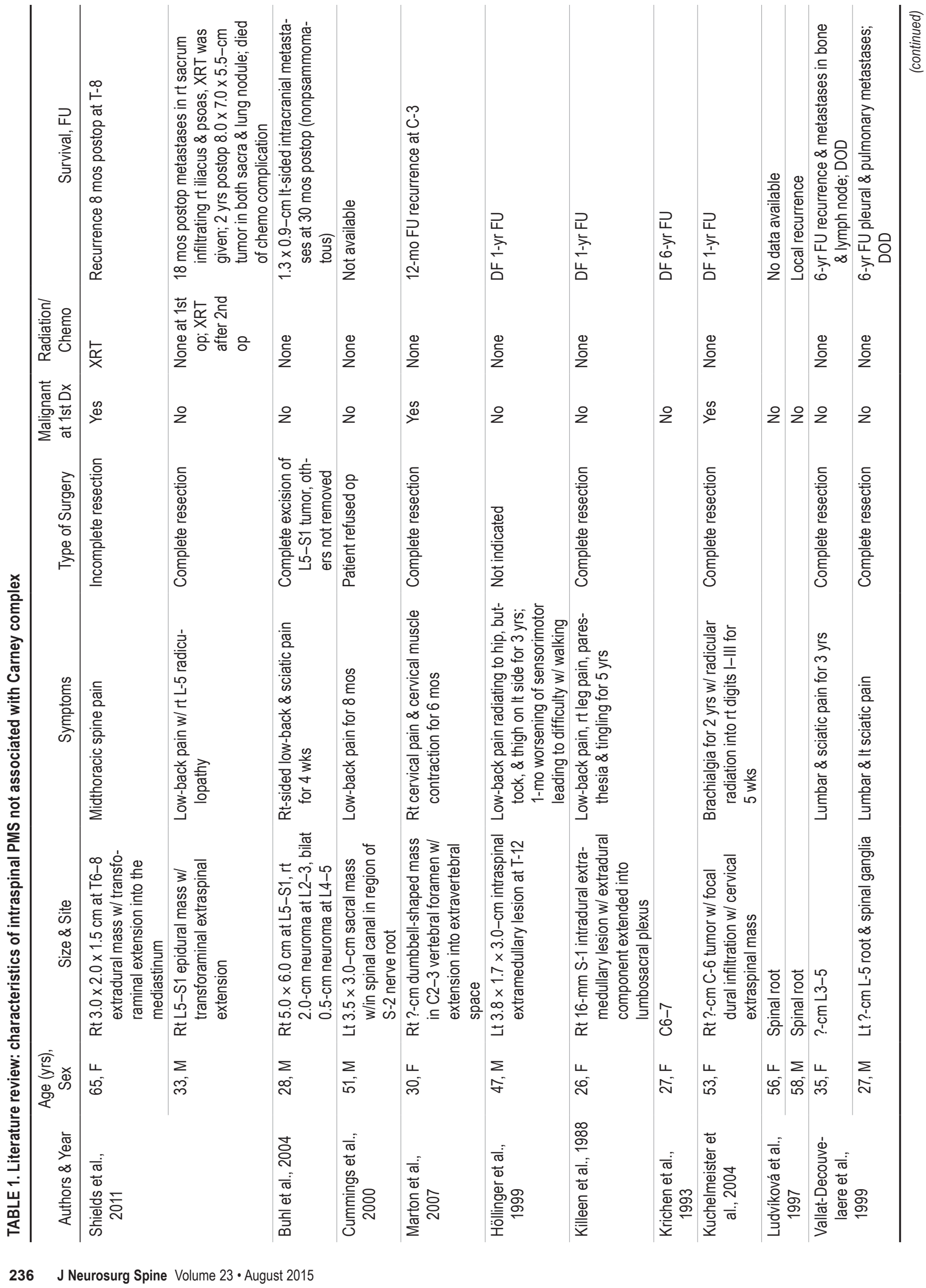




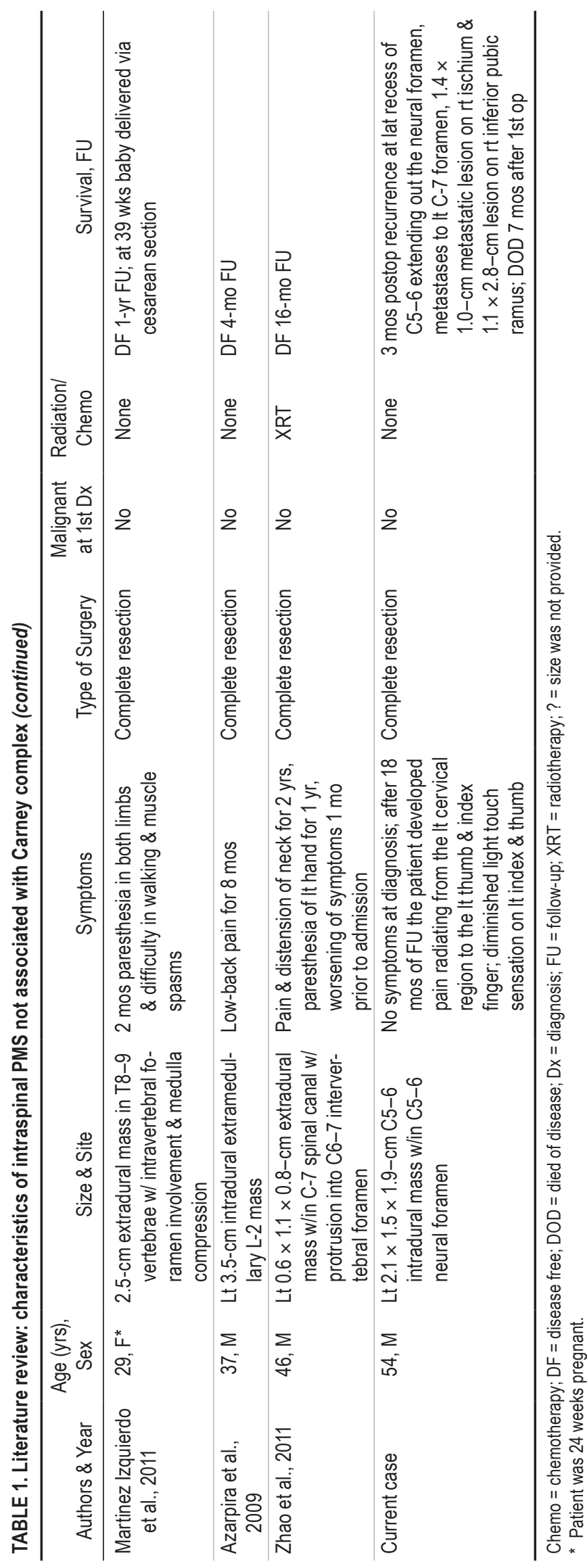

these tumors are different from common schwannomas since PMS contains psammoma bodies, melanin, and adipose tissues. The imaging modality of choice is MRI. The tumor is hyperintense on T1-weighted imaging and isointense on T2-weighted imaging due to free radicals in melanin. ${ }^{7}$

Carney analysis of 40 PMSs from 31 patients showed that 14 cases were not associated with features of the complex. ${ }^{3}$ Those cases that were associated with Carney complex tended to occur in younger patients with an average age of 20 years, while patients without the complex were around 33 years of age. However, the behavior of PMS is not clear since there has not been a single paper that has focused on intraspinal PMS not associated with Carney complex. In a literature review performed by Marton et al. ${ }^{13}$ the authors reported 15 cases of PMS not associated with Carney complex (11 intraspinal and 4 extraspinal). Of the 11 cases of intraspinal PMS, 4 patients had recurrence or metastasis, 3 patients had no follow-up, and 1 patient refused surgery. None of the 4 patients with progression of the disease had histopathological findings of malignant PMS at diagnosis. However, there was 1 patient who had malignant PMS at diagnosis, and the patient was disease free at the 1-year follow-up. The percentage of progression to malignancy in this study was $36.4 \%$ (4 of 11). If we exclude the patients who had no follow-up and the patient who refused surgery, it was $57.1 \%$ (4 of 7).

In our review of the literature (Table 1), we found a total of 17 reported intraspinal PMSs (in 8 females and 9 males; age range 26-65 years, mean 41 years) not associated with Carney complex (including our patient). Of these 17 cases, only 3 were malignant at the time of diagnosis. Of the 3 cases of malignant PMS, one of them developed recurrence within 1 year postsurgery. Of 14 patients who had nonmalignant PMS at the time of diagnosis, 6 developed either recurrence or metastasis, 2 did not have any follow-up, and 7 were disease free at up to 6 years. The percentage of progression to malignancy was $41.1 \%$ ( 7 of 17) in all reported cases of intraspinal PMS and 46.7\% (7 of 15) if we exclude the patient who did not have follow-up and the patient who refused surgery.

Currently, there have not been any reports of the most common type of symptoms or the delay between the first symptoms and the diagnosis for PMS not associated with Carney complex. Based on our results, the most common symptoms were paresthesia and radiculopathy, which over time led to weakness depending on the location of the tumor. The delay between the first symptoms and the diagnosis ranged from 4 weeks to 5 years, and the most common intraspinal tumor site was cervical (5 cervical cases, 3 thoracic, 3 lumbar, 2 sacral, and 2 L5-S1).

The surgical and postsurgical management of PMS not associated with Carney complex have been based on the previous studies of melanotic schwannoma or PMS associated with Carney complex. In our review of literature, 11 of 17 patients had complete resection, 1 patient refused surgery, 1 patient had incomplete resection, and 4 had no available data. Of the 11 patients, 2 had malignant PMS at the diagnosis. Five of 9 patients $(55.6 \%)$ with complete resection with benign PMS developed recurrence, 50\% of patients ( 1 of 2$)$ with complete resection of malignant 
PMS at diagnosis developed recurrence, and $100 \%$ of patients (1 of 1) with incomplete resection of malignant PMS developed recurrence of the tumor. Two patients (one with malignant and one with nonmalignant PMS) underwent radiation treatment postoperatively. The patient with malignant PMS developed recurrence of the tumor within 8 months postoperatively, but the patient without malignant tumor was disease free 16 months postoperatively.

We propose that there can be 2 methods of recurrence and metastasis of PMS: malignant transformation of the tumor or spreading via CSF after violation of subarachnoid space. It has been previously reported that melanotic schwannoma, regardless of the presence of psammoma bodies or Carney complex, has the risk of malignant transformation. ${ }^{13}$ In our patient, the first pathology came back as benign (Fig. 2), but the second pathology was read as malignant (Fig. 4). Although the previously reported cases did not discuss the method of recurrence and metastasis, our report indicates that the possible malignant transformation should be taken into consideration for patients with PMS not associated with Carney complex.

Another possible method that may lead to recurrence of the tumor is seeding via CSF. As previously indicated, our patient's tumor capsule and subarachnoid space were violated. The opening of the tumor capsule and violation of the subarachnoid space may have provided an opportunity for tumor cells to seed the CSF. In our literature review, we tried to determine whether the location of the tumor (i.e., intradural vs extradural) makes a difference in recurrence and metastasis. For accessing the intradural tumor, it is clear that violation of subarachnoid space is necessary and this might cause seeding via CSF. However, the data were limited because not all previous papers indicated the location (i.e., intra- vs extradural) of the tumor.

\section{Conclusions}

Follow-up of patients with PMS not associated with Carney complex must be rigorous and focus not only on the regrowth of the primary tumor but also on metastasis to the distal sites. Patients must be monitored postoperatively for many years since some of the reported patients experienced a recurrence or metastasis after up to 6 years. Recurrence and metastasis can be due to either malignant transformation or CSF seeding. The surgeon should try not to violate the capsule to prevent seeding of the CSF. Currently, the treatment modality of choice is complete resection; however, based on our results, $55.6 \%$ of patients (5 of 9) with benign PMS developed recurrence after complete resection. Further studies are needed to prove the efficacy of radiation therapy and other possible treatment modalities for these patients.

\section{Acknowledgments}

We would like to thank the Cowles Charitable Trust and the Corso Family Charitable Foundation, Inc. for their financial gifts in support of this work.

\section{References}

1. Azarpira N, Torabineghad S, Sepidbakht S, Rakei M, Bagheri $\mathrm{MH}$ : Cytologic findings in pigmented melanotic schwannoma: a case report. Acta Cytol 53:113-115, 2009
2. Buhl R, Barth H, Hugo HH, Mautner VF, Mehdorn HM: Intracranial and spinal melanotic schwannoma in the same patient. J Neurooncol 68:249-254, 2004

3. Carney JA: Psammomatous melanotic schwannoma. A distinctive, heritable tumor with special associations, including cardiac myxoma and the Cushing syndrome. Am J Surg Pathol 14:206-222, 1990

4. Carney JA, Gordon H, Carpenter PC, Shenoy BV, Go VL: The complex of myxomas, spotty pigmentation, and endocrine overactivity. Medicine (Baltimore) 64:270-283, 1985

5. Cummings TJ, Liu K, Jordan LK III, Dodd LG: Fine-needle aspiration diagnosis of psammomatous melanotic schwannoma. Diagn Cytopathol 23:55-58, 2000

6. Hodson JJ: An intra-osseous tumour combination of biological importance-invasion of a melanotic schwannoma by an adamantinoma. J Pathol Bacteriol 82:257-266, 1961

7. Höllinger P, Godoy N, Sturzenegger M: Magnetic resonance imaging findings in isolated spinal psammomatous melanotic schwannoma. J Neurol 246:1100-1102, 1999

8. Killeen RM, Davy CL, Bauserman SC: Melanocytic schwannoma. Cancer 62:174-183, 1988

9. Krichen H, Daghfous MS, Mrabet A, Douik M, Slimane N, Forest M: [Extended melanocytic tumor of the cervical spine. Apropos of a case of melanotic schwannoma.] Ann Pathol 13:184-187, 1993 (Fr)

10. Kuchelmeister K, Lotz C, Schonmayr R, Schachenmayr W: April 2004: woman in her early fifties with a cervical intraspinal and extraspinal mass lesion. Brain Pathol 14:453454, 458-459, 2004

11. Ludvíková M, Michal M, Marek J, Syrůcek M: [Psammomatous melanotic schwannoma.] Cesk Patol 33:141-145, 1997 (Czech)

12. Martinez Izquierdo MA, Lopez-Soto V, Saenz-Santamaria J, Lacruz-Pelea C: Intraoperative cytological findings in two cases of psammomatous melanotic schwannoma. Cytopathology 22:60-62, 2011

13. Marton E, Feletti A, Orvieto E, Longatti P: Dumbbell-shaped C-2 psammomatous melanotic malignant schwannoma. Case report and review of the literature. J Neurosurg Spine 6:591-599, 2007

14. Miller WG: A malignant melanotic tumor of ganglion cells arising from a thoracic sympathetic ganglion. J Pathol Bacteriol 35:351-357, 1932

15. Shields LB, Glassman SD, Raque GH, Shields CB: Malignant psammomatous melanotic schwannoma of the spine: A component of Carney complex. Surg Neurol Int 2:136, 2011

16. Vallat-Decouvelaere AV, Wassef M, Lot G, Catala M, Moussalam M, Caruel N, et al: Spinal melanotic schwannoma: a tumour with poor prognosis. Histopathology 35:558-566, 1999

17. Zhao QH, Zhi S, Wang Z, Tian JW: Psammomatous melanotic schwannoma with cystic changes from old hemorrhages in the cervical spinal canal: a case report. Orthop Surg 3:143-146, 2011

\section{Author Contributions}

Conception and design: Shabani. Acquisition of data: Shabani, Seidman, Davis. Analysis and interpretation of data: Shabani. Drafting the article: Shabani. Critically revising the article: Shabani, Fiore. Reviewed submitted version of manuscript: all authors. Administrative/technical/material support: Fiore. Study supervision: Seidman, Davis.

\section{Correspondence}

Saman Shabani, Department of Neurological Surgery, Medical College of Wisconsin, 9200 W. Wisconsin Ave., Milwaukee, WI 53226. email: sshabani@mcw.edu. 